\title{
Diagnosis telling in people with psychosis
}

\author{
Alyssa C. Milton*12 \& Barbara A. Mullan ${ }^{13}$ \\ ${ }^{1}$ School of Psychology University of Sydney Australia \\ ${ }^{2}$ Division of Psychiatry (Formerly Mental Health Sciences) University College London UK \\ ${ }^{3}$ School of Psychology and Speech Pathology, Curtin University, Australia \\ *Corresponding author: Alyssa Milton School of Psychology University of Sydney NSW 2006 \\ Australia (email: amil2403@uni.sydney.edu.au) \\ Original Publication: This manuscript has not been published in its current form or a \\ substantially similar form (in print or electronically, including on a web site). This manuscript \\ has not been accepted for publication elsewhere, and is not under consideration by another \\ publication.
}

\section{Conflicts of interest: None}

Acknowledgements: With thanks to Dr Kirby Sainsbury (School of Psychology; University of Sydney, Australia). 


\begin{abstract}
Purpose of review: There are complexities in communicating diagnostic information relating to schizophrenia spectrum disorders. There is a current dearth of research in understanding how clinicians effectively communicate with service users about such diagnostic news. In this review we aim to synthesise the latest research throughout 2012 and 2013 that presented data relating to the communication of a diagnosis of schizophrenia spectrum disorders, including individuals who had experienced first episode psychosis or were in an at risk mental states. Comprehensive database and manual searches were conducted which obtained data from both service users and health professional groups.
\end{abstract}

Recent findings: Fourteen quantitative and qualitative studies were found. The majority of studies were descriptive and heterogeneous in content. Key themes included service user preferences towards disclosure and diagnostic terminology, health professional training, stigmarelated issues and the use of diagnostic communication models.

Summary: Overall, communication models that foster therapeutic relationships and actively encourage the health professional to reduce stigma may be a key to initial diagnostic discussions in clinical practice. Such communication models and intervention require further more rigorous evaluation as none have been tested through randomised controlled protocols in clinical settings.

Keywords: Communication, diagnosis, psychosis, schizophrenia, stigma 


\section{Introduction}

Communication between clinicians and mental health service users is at the centre of psychiatric practice and has the ability to influence service users' outcomes including treatment engagement, following treatment suggestions, satisfaction, symptom severity and referral to other services (1). Part of the communication dialogue involves providing information about an individual's diagnostic condition for the first time. Formulating and subsequently communicating diagnostic information relating to schizophrenia spectrum disorders in particular is a complex task. The debate surrounding diagnosis that continued throughout the release of the DSM-V highlighted some challenges to the diagnostic process, such as heterogeneous presentations, a lack of pathogonomic symptoms, fuzzy boundaries with other disorders or the condition's atypical course (2). There are also social reasons for such complexities in formulation and communication of diagnosis. For example, the diagnostic label itself "can vindicate and blame, can legitimise or stigmatise, can facilitate access to resources just as it can restrict opportunities. A diagnosis can be welcomed or eschewed” ((3) p. 797). Psychiatry needs a clear compass for facilitating such discussions with service users; however, there remains a limited understanding in the literature about how best to communicate diagnostic news $(4,5)$.

The advantages and disadvantages of discussing a diagnosis of schizophrenia and related disorders have been considered comprehensively in other literature reviews (6-9). Although providing information to individuals about their diagnosis can require a balance between beneficence and service user autonomy, reviews have formed the general consensus that service users have full rights to information pertaining to their condition and treatment $(4,6,10,11)$. Thus a level of transparency around the diagnosis is recommended; however, the practical 
application of initiating such discussions needs further exploration. Consequently, this paper is concerned with exploring the key ingredients of initially communicating mental health information, and where necessary, a diagnosis. To date the research remains limited. As it is an emerging research area, communication specifically concerned with schizophrenia spectrum disorders including those with first episode psychosis and at risk mental states requires review. This review includes original research from 2012 and 2013 only and discusses it in context with previous research.

\section{Methods}

A systematic search of the databases Embase, Medline, and PsycINFO was undertaken from January 2012 to November 2013 using the search terms: communicat* OR “breaking news” OR tell* OR disclos*, AND “psychosis”* OR schizophrenia OR “schizo-affective”, AND diagnos*. Hand-searching of reference lists and grey literature searches were undertaken on Google Scholar. Quantitative and qualitative studies that presented data describing the provision or receipt of a diagnosis of psychosis or a related condition were included in the review.

\section{Results and Discussion}

The specified search strategy identified 505 citations with duplicates removed; after screening, 14 full-text articles and reviews were considered for this summary paper. The retrieved studies were from four main cohorts: (i) individuals identified as being in an at risk mental state; (ii) individuals who had experienced first episode psychosis; (iii) individuals who had received a schizophrenia spectrum diagnosis; and (iv) mental health professionals. The current research is heterogeneous in terms of its scope; however, key themes that emerged in the literature included 
service user preferences towards disclosure and diagnostic terminology, health professional training, stigma-related issues and diagnostic communication models.

In this review findings for these four main groups are briefly summarised then a subsequent discussion of these key themes are presented.

\section{Studies with individuals with at risk mental states and first episode psychosis}

One review, two survey, and two qualitative studies involving individuals with at risk mental states or first episode were identified (12-16)*. Only one of these studies presented data from individuals with first episode psychosis indicating a dearth of research in this area. Although a formal diagnosis has not yet arisen for those in at risk mental states, the information and communication preferences of this group may prove useful. Despite this, there is a lack of research on communication of risk information in the early intervention field (12)*, and in particular, in exploring service user communication preferences, the ways in which these groups interpret diagnostic denotations, and the extent to which this affects outcomes and behaviour. The available evidence did, however, suggest that individuals want information pertaining to their condition or status $(13,15)^{*}$. Other key findings were that psycho-education is a popular and highly acceptable form of intervention (14)*, and methods for effectively coping with stigma stress could be a key element of support during and after initial diagnostic discussions (16)*.

\section{Studies with individuals with schizophrenia or related disorders}

Three survey studies, one qualitative study, one intervention study and one review have reported results obtained from individuals with a diagnosis of a schizophrenia spectrum disorder $(5,10$, 17-20)*. Descriptive studies highlighted the relationship between an individual’s insight, 
perceived stigma (17)* and level of hope (18)*. One study presented data from a pre-post intervention design that produced promising results for reducing stigma stress stemming from a schizophrenia diagnosis (19). Another survey study described service users’ preferences around diagnostic terminology (20), and qualitative research presented findings on service users preferences for diagnostic discussions (5) which may aid development of diagnostic communication models.

\section{Studies with health professionals}

Three descriptive survey studies published in the 2012 to 2013 period examined views of both medical students $(21,22)^{*}$ and health professionals $(23) *$ These papers highlighted the need to address stigma in both students and psychiatrists.

\section{Information preferences}

Welsh and colleagues explored the information preferences of individuals that were in an at risk mental state or had experienced first episode psychosis (14)*, and found that psycho-education was the most frequently endorsed type of intervention. This indicates that individuals in the early stages of receiving mental health support do want access to information about their mental health and risk situation. Qualitative research by the same group explored five individuals' perspectives on receiving mental health related information concerning their 'at risk' status $(13,15)$, with all participants stating that they wanted to be informed about their risk status; a theme the researchers labelled as: 'it is better to say it'. The 'at risk' label was seen to normalise the prepsychotic experiences and provided reassurance that the condition was treatable, with some finding it refreshing for professionals to be open about their mental health circumstances. Withholding information was seen as potentially more harmful over the longer term. 
The DSM-V has triggered much debate about diagnostic terminology particularly in relation to schizophrenia; however, only one study in the past two years has examined language preferences of people with a schizophrenia spectrum diagnosis (20)* This small qualitative study compared the term "schizophrenia" to the potential replacement classification of "salience syndrome". Results indicated that service users' preferences were mixed. Although it was slightly more popular, "salience syndrome” was seen as difficult to relate to or understand. A significant link was found between the diagnosis individuals self-identified as having and their preferred labelling terminology, such that individuals who preferred the label 'schizophrenia' also initially self-identified with the diagnosis. Research prior to 2012 recommended that service users have a right to diagnostic information $(4,6,10,11)$; however, reactions to diagnostic news vary between service users $(5,11,24)$. Therefore, an individualized approach to diagnostic discussion may be required and how individuals interpret diagnostic information and apply language to describe their condition could be choice based.

\section{Relational aspects of the diagnostic discussion}

Service users place value on the relationships and opportunities for learning through contact with both clinicians and fellow service users $(5,13,15)^{*}$. Findings suggested that clinicians' personal qualities are paramount during a diagnostic conversation (5)*. These including listening to the individual, taking a non-judgmental approach, fostering empathy, honesty, mutual respect, and possessing a good mental health knowledge base. Transparent information sharing, conveying hopeful information, utilising collaborative approaches, and actively addressing stigma also were highlighted as vital to the diagnostic communication process. These suggestions appear in line with what constitutes good therapeutic practice. For example, in a recent conceptual review, Priebe and colleagues (25) suggested five guiding principles for good communication in 
psychiatry: a focus on the individual's concerns, positive regard and personal respect, appropriate involvement of service users in decision making, genuineness with an individualised and personal touch, and the use of a psychological treatment model.

\section{The need for health professional and student training}

Although the incidence of people being told information pertaining to their diagnosis has

increased, schizophrenia remains one of the least disclosed mental health conditions (10)*. There may be a specific need for health professional to have support and training around providing such information with service users. For example, in 2001 Clafferty and colleagues (8) reported that a large proportion of psychiatrists in Scotland felt uncomfortable telling service users a schizophrenia diagnosis (43\%), whereas few indicated that they thought services users would not want to know their diagnosis (13\%). Overall, health professionals appear to find initially discussing a diagnosis with a service user challenging, and when asked, indicate a desire for specific training to learn effective methods for discussing diagnostic news (26). Previous reviews have also highlighted the need for a greater focus on clinician communication training in this area $(4,9,10)$.

Two studies in the 2012 to 2013 period examined medical students' views on diagnostic discussions $(21,22) *$. Compared to first year students, those in their later years of study were less likely to agree that users' should be informed about their own condition or about medications and their side effects. The researchers argued this was due to more advanced students having developed prejudices about people with schizophrenia, having a less transparent view on information sharing, and exhibiting reduced confidence regarding service users 
participating in psychological treatments. In other research from the same group it was reported that more medical education resulted in greater stigma $(21)^{*}$, leading to the conclusion that addressing stigma needs to be on the educational agenda for students. This may also need addressing in clinicians, as Loch and colleagues have reported that psychiatrists are not a homogenous group in relation to their level of stigma towards people with schizophrenia, which impacts upon their treatment practices (23)*.

\section{Stigma and interventions}

Stigma-related issues are a chief barrier to communication and can heavily influence service users' subsequent reaction to diagnostic information $(4,10,11)$. Over the last two years, the issue of stigma was explored in six studies (13, 15-19)*, two of which explored individuals' perspectives on receiving mental health-related information concerning an 'at risk' status using a qualitative methodology $(13,15)^{*}$. Specifically, it was found that stigma was associated with anxiety, although few participants had actually experienced negative stigma and most had experienced acceptance and support. This appears to suggest that although stigma stress does exist for individuals in the early stages of diagnostic communication and engagement with mental health services, it may be negated with appropriate support. Other research to support this hypothesis has been reported in survey studies; for example, the perceived public stigma, shame, and self-labelling of individuals with at risk mental states and first episode psychosis were all found to be independently associated with an increase in stigma stress (16)*. For those who have received a diagnosis of schizophrenia, there have also been correlations found between level of insight and self-reported stigma (17)*. The authors suggested that this might be indicative of a motivational pathway to insight formation. Negative concepts of illness, which may be produced by the stigmatization process, may also predict negative outcomes for individuals. Similar to the 
recommendations from Rusch and colleagues for individuals with first episode psychosis and at risk mental states (16)*, Pruß’ research group suggests that future interventions should be tailored to the subjective understanding of the individual and aim to address stigma concepts.

Research has provided some further guidance on how interventions might be tailored to help individuals respond to stigma related concerns during the diagnostic process (18)*. Schrank and colleagues found a strong causal relationship between hope, depression and self-stigma, with insight being found to diminish hope and increase depression and self-stigma (18)*. Again, the authors suggested that interventions should be individualised and should specifically aim to increase levels of hope in order to reduce self-stigma. A small scale non-randomised intervention that was developed by Sibitz and colleagues (19)* to address internalized self-stigma was found to be successful, such that intervention participants showed a significant reduction in internalized stigma and greater improvement in the quality of life and overall psychopathology. These results are encouraging and show that such interventions may complement therapeutic intervention. They do, however, need to be evaluated through randomized controlled designs.

\section{Models for communicating news}

Reviews have called for diagnostic communication models to be developed from the evidence base in order to support the individual and the clinician effectively through the diagnostic communication process $(4,9,10)$. Levin and colleagues (27) utilised an online forum with input from psychiatrists to develop a model of delivering diagnostic news of schizophrenia. Key steps to aid this process were produced, including preparing for the meeting, negotiating the agenda collaboratively, reviewing the consumers and families understanding of schizophrenia, 
discussing the prognosis, discussing meaning of schizophrenia whilst promoting a recovery framework and effectively planning follow-up. An alternative model of communication is the SPIKES protocol, which is a stepwise process for breaking difficult news borrowed from oncology (28). The basic framework for the SPIKES protocol consists of six steps: Setting up the interview; assessing the individual's Perception of the medical circumstances; obtaining the person’s Invitation to receive the information; giving the requisite Knowledge; responding Empathically to the emotions as a consequence of the news; and Summarize the treatment processes. These models of communication may prove useful; however, neither has been empirically tested in the mental health field.

The SPIKES protocol is the most frequently referenced model for disclosing diagnostic news in mental health $(4,9,10)$. The only research in 2012 to 2013 to look service users' preferences towards the SPIKES models was qualitative research by Milton and Mullan (5)*. Service users' views of what constitutes good communication during diagnostic discussions were found to correspond to the stepwise framework of the SPIKES protocol. The model for communication may prove to be a useful guide to initial diagnostic discussions; however, it could need expansion to be fully appropriated into a mental health context (5)*. This is particularly because communication of diagnostic information may be delivered over a number of support sessions depending on the individuals needs, and because stigma related issues may need specific intervention.

\section{Future directions}

A key conceptual issue in communication research raised by McCabe and Priebe is that there is no definitive model of 'good communication' (1), this issue can also be applied to the initial 
stage of diagnostic communication. With the recent development of potential communication models that outline methods for initiating preliminary diagnostic discussions, there is now a need for rigorous evaluation. This also is essential for interventions that aim to reduce stigma stress. It is acknowledged that communication is both challenging and cumbersome to study (1), however pragmatic and sufficiently large studies are necessary using randomised controlled trial protocols.

\section{Conclusions}

For individuals receiving news of a diagnosis of schizophrenia or related disorders, there is evidence to suggest individuals want information and psycho-education is highly acceptable as part of the initial diagnostic discussion. The therapeutic relationship is highly important as is involvement of service users fully in the communication process. Stigma reduction appears vital in the discussion as it may be a key element of insight formation, and stigma stress impacts negatively upon service users' outcomes. Preliminary interventions to reduce stigma stress and increase levels of hope present some promising results but need more rigorous evaluation. Both mental health professionals and students also report stigma which can impact on their beliefs and practice. Communication models that foster therapeutic relationships and actively encourage the health professional to reduce stigma may be a key to initial diagnostic discussions and may be a necessary adjunct to communication training.

\section{Key Points:}

- Given the incidence of mental health diagnosis, there is a current lack of research on how health professionals initially communicate diagnostic information or a formal diagnosis to service users. 
- The limited research in this area highlights that service users want information, particularly psycho-education; however, stigma stress can arise as part of developing insight about a diagnosis.

- Interventions to address stigma stress and increase hope in service users show promise, but need further evaluation.

- The clinician-service user relationship is important, which includes information sharing, conveying hopeful information, utilising collaborative approaches and actively addressing stigma.

- As research suggests that diagnostic conversations can be challenging for clinicians, models of diagnostic communication might aid clinician training and ultimately the diagnostic conversation with service users. 


\section{References}

1. McCabe R, Priebe S. Communication and psychosis: it's good to talk, but how? British J Psychiat. 2008;192(6):404-5.

2. Frances A. Essentials of psychiatric diagnosis: Guilford Publication; 2013.

3. Jutel A, Nettleton S. Towards a sociology of diagnosis: Reflections and opportunities. Social Science \& Medicine. 2011;73(6):793-800.

4. Cleary M, Hunt GE, Horsfall J. Delivering difficult news in psychiatric settings. Harvard Review of Psychiatry. 2009;17(5):315-21.

5. *Milton A, Mullan B, editors. Utilising inductive approaches and service-user consultation to develop a model for diagnostic communication in mental health. Psychology \& Health; 2013: Taylor \& Francis LTD, Oxon, England. Qualitative research that is the first to examine service user preferences towards disclosure to inform models of diagnostic communication.

6. Atkinson J. To tell or not to tell the diagnosis of schizophrenia. J Med Ethics. 1989;15(1):21-4.

7. Donnelly P. To tell or not to tell the diagnosis of schizophrenia. Journal of medical ethics. 1989;15(3):166.

8. Clafferty R, McCabe E, Brown K. Conspiracy of silence? Telling patients with schizophrenia their diagnosis. Psychiatr Bull. 2001;25(9):336-9.

9. Seeman M. Breaking Bad News: Schizophrenia. Journal of Psychiatric Practice. 2010;16(4):26976.

10. Milton A, Mullan B. The communication of mental health diagnosis: A Systematic Review. BPS DHP Annual Conference; 5 - 7th September; Liverpool, UK.2012.

11. Rose D, Thornicroft G. Service user perspectives on the impact of a mental illness diagnosis. Epidemiol Psichiatr Soc. 2010;19(2):140-7. Epub 2010/09/08.

12. *Welsh P, Tiffin P. Attitudes of patients and clinicians in relation to the at-risk state for psychosis. Early Intervention in Psychiatry. 2013:A narrative review that highlights the dearth of communcation research in diagnosis in the early intervention field.

13. *Welsh P, Tiffin P. Observations of a small sample of adolescents experiencing an at-risk mental state (ARMS) for psychosis. Schizophrenia Bulletin. 2012;38(2):215-8. This study explored how adolescents with an ARMS label understand and experience their condition medically and personally.

14. *Welsh P, Tiffin P. Assessing adolescent preference in the treatment of first-episode psychosis and psychosis risk. Early Intervention in Psychiatry. 2013:This study investigated the treatment preferences of adolescents with a first-episode psychosis or at-risk mental state for psychosis. The majority endorsed psychoeducational material as the most popular treatment choice (36/40; 90\%). .

15. *Welsh P, Brown S. 'I'm not insane, my mother had me tested': the risk and benefits of being labelled 'at-risk' for psychosis. Health, Risk \& Society. 2013:1-15. This article qualitatively explores young people deemed to be at an elevated risk of developing psychosis using an Interpretative Phenomenological Analysis framework. Three key themes were identified: 'It is better to say it', 'How others would take me' and 'Just to have somebody to talk to'.

16. *Rüsch N, Heekeren K, Theodoridou A, Dvorsky D, Müller M, Paust T, et al. Attitudes towards help-seeking and stigma among young people at risk for psychosis. Psychiatry Research. 2013:This paper presents finings that suggest that for young people at risk for psychosis, stronger self-labeling and less stigma stress predicted better attitudes towards psychiatric medication and psychotherapy.

17. *Pruß L, Wiedl KH, Waldorf M. Stigma as a predictor of insight in schizophrenia. Psychiatry Research. 2012;198(2):187-93. This study indicates significant association of variability in insight with stigma constructs, which may indicate a motivational pathway of insight formation.

18. *Schrank B, Amering M, Hay AG, Weber M, Sibitz I. Insight, positive and negative symptoms, hope, depression and self-stigma: a comprehensive model of mutual influences in schizophrenia 
spectrum disorders. Epidemiology and psychiatric sciences. 2013:1-9. This study reported strong and mutual causal influences between hope, depression and self-stigma for individuals with schizophrenia. . 19. *Sibitz I, Provaznikova K, Lipp M, Lakeman R, Amering M. The impact of recovery-oriented day clinic treatment on internalized stigma: Preliminary report. Psychiatry Research. 2013:This study showed significant postive results for an intervention aimed at addressing internalised stigma for individuals with schizophrenia using controlled pre-post test design.

20. *Tranulis C, Lecomte T, El-Khoury B, Lavarenne A, Brodeur-Côté D. Changing the Name of Schizophrenia: Patient Perspectives and Implications for DSM-V. PLoS ONE. 2013;8(2):e55998. This was the first study to explore both quantitatively and qualitatively the effects on stigma based on diagnostic labels of 'schizophrenia' and 'salience syndrome'.

21. *Magliano L, Read J, Sagliocchi A, Patalano M, D'Ambrosio A, Oliviero N. Differences in views of schizophrenia during medical education: a comparative study of 1st versus 5th-6th year Italian medical students. Social Psychiatry and Psychiatric Epidemiology. 2012:1-9. This paper presents data indicating a need to include education on stigma and recovery in schizophrenia in the training of medical students.

22. *Magliano L, Read J, Sagliocchi A, Patalano M, Oliviero N. Effect of diagnostic labeling and causal explanations on medical students' views about treatments for psychosis and the need to share information with service users. Psychiatry Research. 2013:This paper presents data that suggests that compared to first year students, those at their fifth/sixth-year of studies more strongly endorsed drugs, had less confidence in psychologists and family support, and were less keen to share information with individuals with schizophrenia.

23. *Loch A, Guarniero F, Lawson F, Hengartner M, Rössler W, Gattaz W, et al. Stigma toward schizophrenia: do all psychiatrists behave the same? Latent profile analysis of a national sample of psychiatrists in Brazil. BMC Psychiatry. 2013;13(1):92. This paper presents data suggesting that psychiatrists are not an homogenous group in terms of their level of stigma towards individuals with schizophrenia.

24. Gallagher A, Arber A, Chaplin R, Quirk A. Service users' experience of receiving bad news about their mental health. J Ment Health. 2010;19(1):34-42.

25. Priebe S, Dimic S, Wildgrube C, Jankovic J, Cushing A, McCabe R. Good communication in psychiatry-a conceptual review. European Psychiatry. 2011;26(7):403-7.

26. Cleary M, Hunt G, Walter G. Delivering difficult news. Views of mental health staff in inpatient settings. J Psychosoc Nurs Ment Health Serv. 2010;48(6):32-9.

27. Levin $T$, Kelly B, Cohen M, Vamos M, Landa Y, Bylund C. Using a psychiatry e-list to develop a model for discussing a schizophrenia diagnosis. Psychiat Serv. 2011;62(3):244-6.

28. Baile WF, Buckman R, Lenzi R, Glober G, Beale EA, Kudelka AP. SPIKES-a six-step protocol for delivering bad news: application to the patient with cancer. The oncologist. 2000;5(4):302-11. 